\title{
Comparison of phytosterol intake from FFQ with repeated 24-h dietary recalls of the Adventist Health Study-2 calibration sub-study
}

\author{
Rawiwan Sirirat ${ }^{1}$, Celine Heskey ${ }^{2}$, Ella Haddad ${ }^{1}$, Yessenia Tantamango-Bartley ${ }^{3}$, Gary Fraser $^{1}$, \\ Andrew Mashchak ${ }^{1}$ and Karen Jaceldo-Siegl ${ }^{1 *}$ \\ ${ }^{1}$ Adventist Health Studies, School of Public Health, Loma Linda University, 24951 North Circle Drive, Nichol Hall 2031, \\ Loma Linda, CA 92350, USA \\ ${ }^{2}$ Center for Nutrition, Healthy, Lifestyle, and Disease Prevention, School of Public Health, Loma Linda University, 24951 North \\ Circle Drive, Nichol Hall 1315, Loma Linda, CA 92350, USA \\ ${ }^{3}$ Global Patient Safety $\&$ Labeling, Nephrology and Inflammation TherapeuticArea, Amgen Incorporation, One Amgen Center \\ Drive, Thousand Oaks, CA 91320, USA
}

(Submitted 16 July 2018 - Final revision received 28 January 2019 - Accepted 27 February 2019 - First published online 28 June 2019)

\section{Abstract}

We evaluated the performance of an FFQ in estimating phytosterol intake against multiple 24-h dietary recalls (24HDR) using data from 1011 participants of the calibration sub-study of the Adventist Health Study-2 (AHS-2) cohort. Dietary assessments of phytosterol intake included a self-administered FFQ and six 24HDR and plasma sterols. Plasma sterols were determined using the GLC flame ionisation method. Validation of energy-adjusted phytosterol intake from the FFQ with $24 \mathrm{HDR}$ was conducted by calculating crude, unadjusted, partial and de-attenuated correlation coefficients $(r)$ and cross-classification by race. On average, total phytosterol intake from the FFQ was $439.6 \mathrm{mg} / \mathrm{d}$ in blacks and $417.9 \mathrm{mg} / \mathrm{d}$ in whites. From the $24 \mathrm{HDR}$, these were $295.6 \mathrm{mg} / \mathrm{d}$ in blacks and $351.4 \mathrm{mg} / \mathrm{d}$ in whites. Intake estimates of $\beta$-sitosterol, stigmasterol, other plant sterols and total phytosterols from the FFQ had moderate to strong correlations with estimates from 24HDR $(r 0 \cdot 41-0 \cdot 73)$. Correlations were slightly higher in whites $(r 0.42-0.73)$ than in blacks $(r 0.41-0.67)$. FFQ estimates were poorly correlated with plasma sterols as well as 24 HDR $v$. plasma sterols. We conclude that the AHS-2 FFQ provided reasonable estimates of phytosterol intake and may be used in future studies relating phytosterol intake and disease outcomes.

Key words: Validation: Dietary assessment tools: Correlation coefficients: Phytosterols

Phytosterols are the phytochemicals that are found to have a structure comparable to cholesterol ${ }^{(1)}$. They are found in plant foods where they function as part of the plant cell membrane ${ }^{(2)}$. There are various types of phytosterol widely grouped into plant sterols and plant stanols. The most abundant phytosterols are $\beta$-sitosterol, stigmasterol and campesterol ${ }^{(3)}$. The main sources of plant sterols are vegetable oils, nuts and seeds ${ }^{(4)}$. Plant stanols are a subgroup of phytosterols that are saturated $^{(3)}$. Plant stanols are found in mixtures of extracted sterols, which is the mixture of free sterols and stanols and their esters. Enriched extracted sterols are found mostly in commercial products such as margarine, fermented milk drinks, salad dressing, spreads, milk, soya, yogurt, cheesy products, soya and fruit drinks, sausages and breads, ready-to-eat meals, snack bars and candies ${ }^{(5)}$.
Dietary intake of plant sterols varies greatly in Western countries. The median phytosterol intake in the European Perspective Investigation into Cancer and Nutrition Spanish cohort is approximately $315 \mathrm{mg} / \mathrm{d}^{(6)}$. The average intake of phytosterols in the UK is $163 \mathrm{mg} / \mathrm{d}^{(7)}$. Phytosterol intake in the usual Spanish diet is approximately $276 \mathrm{mg} / \mathrm{d}^{(8)}$.

The cholesterol-lowering property of phytosterols is one of the well-established health benefits of plant sterols and plant stanols. For example, it has been shown that an intake of $2 \mathrm{~g} / \mathrm{d}$ of stanols or plant sterols lowers plasma LDL-cholesterol levels by approximately $10 \%{ }^{(9)}$. Plant sterols and stanols also have anticancer properties ${ }^{(10)}$.

Phytosterol intake is difficult to assess due to the lack of comprehensive updated plant sterol and stanol composition data, particularly related to plant stanols in fortified foods. Of the

Abbreviations: 24HDR, 24-h dietary recall; AHS-2, Adventist Health Study-2; USDA SR, US Department of Agriculture National Nutrient Database for Standard Reference.

* Corresponding author: Karen Jaceldo-Siegl, email kjaceldo@llu.edu 
published reports on phytosterol intake to date, the most comprehensive and referenced article dates back to $1978^{(11)}$. To our knowledge, only one validation study on phytosterol consumption was conducted in 2013 by Northern Sweden group whom found the moderate to high association between FFQ and 24-h dietary recall (24HDR) ${ }^{(12)}$

We estimated phytosterol intake in the Adventist Health Study-2 (AHS-2) population, which is a prospective cohort of adult Adventists in North America, with a wide range of plant foods intake ${ }^{(13)}$. AHS-2 participants are $48.2 \%$ non-vegetarian, $5.5 \%$ semi-vegetarian, $9.8 \%$ pesco-vegetarian, $28.9 \%$ lacto-ovo vegetarian and $7 \cdot 6 \%$ vegan $^{(14)}$. The primary dietary assessment method in the AHS-2 is the FFQ, a widely used approach to assess habitual dietary intake of large study populations ${ }^{(15)}$. In order to further associate dietary intake (based on FFQ) with disease outcomes, it is crucial to first examine the performance of the FFQ in measuring true intake. In the AHS-2, a calibration sub-study was conducted for the purpose of validating food frequency data and to correct biases related to measurement errors $^{(13)}$.

The objective of this paper is to compare plant sterol and plant stanol intake assessed by the FFQ intake with multiple $24 \mathrm{HDR}$ as the reference, using data from the calibration substudy of the AHS-2.

\section{Methods}

\section{Study design}

The AHS-2 is a prospective cohort of 95873 adults. Baseline data collection was from 2002 to 2007. Participants of this cohort had to be 30 years or older and sufficiently fluent in English in order to complete a comprehensive lifestyle questionnaire which included the $\mathrm{FFQ}^{(13)}$. In order to validate the dietary information of the comprehensive lifestyle questionnaire, the investigators of AHS-2 conducted a calibration sub-study of 1011 subjects from the AHS-2 cohort. Calibration sub-study subjects were randomly selected by church location, and then subjects within each church were selected by sex and age. Black participants were purposefully oversampled to ensure more similar proportions of black and white participants. Throughout the 9- to 12-month period of the calibration study, the data collection included the FFQ, six 24HDR and collection of biological specimens (i.e. plasma, serum, urine, etc.).

We excluded subjects who did not complete the requisite number of recalls ( $n$ 96), or subjects with an incomplete FFQ ( $n$ 34), total energy intake greater than $4500 \mathrm{kcal}(18830 \mathrm{~kJ})$ or less than $500 \mathrm{kcal}(2090 \mathrm{~kJ})$ and/or a BMI greater than 50 or less than $15 \mathrm{~kg} / \mathrm{m}^{2}$ ( $n$ 102). After these exclusions, the number of participants available for statistical analysis was 779 . The analytic subjects and those who were excluded from the analysis were found to be similar in baseline characteristics.

This study was conducted according to the guidelines laid down in the Declaration of Helsinki, and all procedures involving human subjects were approved by the institutional review board of Loma Linda University (institutional review board no. 48134). Written informed consent was obtained from all subjects.

\section{Dietary assessments}

FFQ. The AHS-2 FFQ is the largest portion of the comprehensive enrolment questionnaire which consists of 204 foods with fiftyfour questions pertaining to food preparation and forty-six openended questions ${ }^{(1)}$. Frequencies are categorised into never or rarely, 1-3 times per month, 1 time per week, 2-4 times per week, 5-6 times per week, 1 time per d, 2-3 times per d, $4+$ times per $\mathrm{d}$ and $6+$ times per $\mathrm{d}$, which were weighted $0,0 \cdot 067,0 \cdot 143$, $0.429,0.786,1,2.5,4.5$ and 6.5 in terms of times per $\mathrm{d}$, respectively. The amount of food consumption was categorised into one standard serving size, half or less and one-and-a-half or more of a standard serving size, and weighted 1, 0.5 and 1.5, respectively ${ }^{(16)}$

24- $h$ dietary recall. We used multiple $24 \mathrm{HDR}$ as the reference method which were obtained over the telephone and without prior announcement ${ }^{(16)}$. Participants were sent a two-dimensional food portion visual to help estimate portion size. Each 24HDR was conducted by a trained research dietitian who asked specific details about food preparation and recipes. These 24HDR were digitally recorded and entered into the Nutrition Data System for Research (NDS-R) version 4.06 or 5.0 (The Nutrition Coordinating Center), and nutrient composition was calculated based on the NDS-R 2008 database. Quality control of the recalls was performed by a senior research dietitian who listened to randomly selected recorded interviews, verified and compared the audio data with the actual entries on the NDS-R database ${ }^{(16)}$.

Two sets of 24HDR were obtained approximately 6 months apart; each set included one Saturday, one Sunday and one weekday, with a total of six $24 \mathrm{HDR}$ per participant. Using one set of the 24HDR, a synthetic week was created using the following formula: (Saturday intake + Sunday intake $+5 \times$ weekday intake) divided by $7 \mathrm{~d}$. Thus the two sets of $24 \mathrm{HDR}$ provided two synthetic weeks of intake data. To estimate the average food intake of each participant in each of the $24 \mathrm{HDR}$, we averaged their phytosterol intake over these two approximated weeks ${ }^{(16)}$.

Phytosterol database. The US Department of Agriculture (USDA) National Nutrient Database for Standard Reference (USDA SR) is produced by the USDA, which is the primary database source of food composition data in the $\mathrm{USA}^{(4)}$. For the present study, we used the USDA SR 27 (August 2014) as the primary source of standard phytosterol contents of over 500 food items.

Throughout this paper, 'plant sterols' refers to $\beta$-sitosterol, campesterol and stigmasterol; 'other phytosterols' refers to $\Delta 5+\Delta 7$ avenasterols, avenasterol, brassicasterol, stanols, stigmastanol, sitostanol, campestanol and other unknown sterols. 'Total phytosterols' refers to plant sterols and other phytosterols combined.

For unavailable foods and ingredients ( $n$ 189) in the USDA SR 27 , we used the phytosterol content which were quantified by the GC method ${ }^{(5,8,11,12,17-25)}$ or GC-MS ${ }^{(26)}$. This particular method was used to quantify phytosterol content in the USDA SR $27^{(27)}$.

Our compiled phytosterol database was comprised of plant sterols, other phytosterols and total phytosterols. Phytosterol 
Table 1. Phytosterol food groups and their components

\begin{tabular}{|c|c|}
\hline Phytosterol food groups & Components \\
\hline Nuts and seeds & $\begin{array}{l}\text { Almonds, cashews, flax seeds, nuts, seeds, } \\
\text { walnuts, tree nuts, trail nuts }\end{array}$ \\
\hline Legumes and soya & $\begin{array}{l}\text { Legumes, peanuts butter, peanuts, } \\
\text { soya beans, tofu }\end{array}$ \\
\hline Vegetables & $\begin{array}{l}\text { Fried potatoes, leafy greens, onions, } \\
\text { other vegetables, potatoes, vegetables, } \\
\text { green beans }\end{array}$ \\
\hline Grains & $\begin{array}{l}\text { Whole grains, refined grains, mixed grains, } \\
\text { refined cereals, mixed cereals }\end{array}$ \\
\hline Oils and added fats & $\begin{array}{l}\text { Added fats and liquid fats: margarine spread } \\
\text { or stick with vegetable oil or soybean oil, } \\
\text { almond oil, rapeseed oil, cocoa butter oil, } \\
\text { coconut oil, maize and rapeseed oil, maize } \\
\text { oil, cottonseed oil, flaxseed oil, grape seed } \\
\text { oil, hazelnut oil, palm oil, peanut oil, nutmeg } \\
\text { butter oil, poppy seed oil, rice bran oil, } \\
\text { safflower oil, sesame oil, shea nut oil, } \\
\text { soybean oil, sunflower oil, tea seed oil, } \\
\text { tomato seed oil, vegetable oil, walnut oil, } \\
\text { wheat germ oil, olive oil }\end{array}$ \\
\hline Olives and & Olives and avocados \\
\hline Fruits & Berries, dried fruits, fruits, fruit juice \\
\hline
\end{tabular}

content was quantified as $\mathrm{mg} / 100 \mathrm{~g}$ from each food item. Once we were able to identify phytosterol content in foods, we quantified phytosterol content based on the FFQ and 24HDR by matching the food ID in the compiled phytosterol database with the food ID and food description in the FFQ and 24HDR of the calibration sub-study.

We also grouped plant foods as sources of phytosterols as follows: nuts and seeds, legumes and soya, vegetables, grains, oils and added fats, olives and avocados and fruits (Table 1).

Phytosterol intake. We determined the 24HDR plant sterol and other phytosterol intake of individual subjects by using the following formula: $\sum C_{n} \times K_{n}$ where $C=$ the reported grams of food $_{n}$ consumed and $K=$ mg of phytosterol content per $100 \mathrm{~g}$ of $\operatorname{food}_{n}$.

Phytosterol intake estimates (mg) from the FFQ were obtained by $\sum F_{n} \times S_{n} \times G_{n} \times K_{n}$, where $F=$ the weighted frequency of food intake ${ }_{n}, S=$ weighted serving size of food consumed $_{n}, G=$ the standard serving size of food $_{n}$ and $K=\mathrm{mg}$ of phytosterol content per $100 \mathrm{~g}$ of food $_{n}$

\section{Laboratory methods}

Blood was collected from participants during clinic visits between first and second 24HDR. Blood processing followed a standard protocol ${ }^{(28)}$. Plasma was derived from blood collected in heparin tubes. Collected blood was separated into layers by centrifuge, and then aliquots of plasma were separated into straws. These sealed plasma straws were put into containers and kept in liquid $\mathrm{N}_{2}$ tanks at the temperature of $-182^{\circ} \mathrm{C}^{(13)}$.

One of these plasma straws from each participant was used for the determination of plant sterol and cholesterol concentration. The concentrations of $\beta$-sitosterol, campesterol and cholesterol were measured using the GLC flame ionisation detection method $^{(29)}$. Plasma samples were sent to the Institute of Clinical Chemistry and Clinical Pharmacology, University
Clinics of Bonn, Bonn, Germany for quantifying plasma sterol and cholesterol concentration.

\section{Statistical analysis}

Prior to analysis, we applied $\log (x+1)$ to variables with zero phytosterol intake ( $n 8$ which represent less than $1 \%$ of the analytic sample). After transformation, the distribution of these phytosterols and other transformed variables was greatly improved and the four usual statistical assumptions (normality, homogeneity of variance, linearity and independence) were met.

All phytosterol intake levels from the FFQ and 24HDR were energy-adjusted using the residual method ${ }^{(15)}$, in order to obtain phytosterol intake without the undue influence of total energy intake. Due to the fact that some individuals had phytosterol intake and few did not, we applied a partitioning method ${ }^{(30)}$. This method allowed us to retain zero intakes and only energy adjusted the non-zero intakes. We then combined energyadjusted non-zero intake levels with the zero intakes, thus keeping all values on the same scale.

Previous reports on the calibration sub-study showed differences in nutrient and food intake by race and no distinct patterns by sex. Therefore, we stratified by race in the analysis of this paper.

Comparison of baseline characteristics by race was done using the independent $t$ test for continuous and chi square for categorical variables. Untransformed phytosterol intake determined from the FFQ, 24HDR and plasma were presented as arithmetic means and standard deviations.

Unadjusted Pearson correlations of the transformed energyadjusted plant sterols, other phytosterols and total phytosterol intake between FFQ and 24HDR were first determined. De-attenuation correlation coefficient determination was then conducted to correct for within-person variation of the $24 \mathrm{HDR}$ prior to correlation with the FFQ.

Contingency tables (cross classification) between the FFQ and 24HDR data, stratified by race, were also produced to determine the agreement between the FFQ and $24 \mathrm{HDR}$ reporting methods. These provided the quantitative differences of the phytosterol intake of the two dietary measurements in a categorical manner ${ }^{(15)}$.

Additionally, we calculated the contribution percentage of each food group to total phytosterol intake levels assessing by FFQ of the calibration sub-study participants. All analyses were done using SAS, version 9.4 (SAS Institute, Inc.)

\section{Results}

Selected characteristics of the calibration sub-study participants by race are shown in Table 2. Age, sex, BMI and energy intakes were statistically significantly different between blacks and whites. Therefore, we further conducted analysis stratified by race. In general, intake of individual plant sterols and total phytosterols was higher when assessed by FFQ than 24HDR in both races. The mean estimated intake of energy-adjusted total phytosterols was $295.6 \mathrm{mg} / \mathrm{d}$ in blacks and $351.4 \mathrm{mg} / \mathrm{d}$ in whites from six 24HDR. Using the FFQ, energy-adjusted total phytosterols was estimated to be $439.6 \mathrm{mg} / \mathrm{d}$ in blacks and $417.9 \mathrm{mg} / \mathrm{d}$ in whites. 
Table 2. Subjects characteristics by race in the Adventist Health Study-2 calibration sub-study ( $n$ 781)

(Mean values and standard deviations; percentages)

\begin{tabular}{|c|c|c|c|c|}
\hline \multirow[b]{2}{*}{ Baseline characteristics } & \multicolumn{2}{|c|}{ Blacks ( $n$ 339) } & \multicolumn{2}{|c|}{ Whites ( $n$ 442) } \\
\hline & Mean & SD & Mean & SD \\
\hline Age (years) & 58.56 & $12 \cdot 80$ & $62 \cdot 37^{*}$ & 13 \\
\hline \multicolumn{5}{|l|}{ Sex } \\
\hline Females (\%) & \multirow{2}{*}{\multicolumn{2}{|c|}{$\begin{array}{l}69.91 \\
30.09\end{array}$}} & \multicolumn{2}{|c|}{$63 \cdot 12^{*}$} \\
\hline Males (\%) & & & \multicolumn{2}{|c|}{$36 \cdot 88$} \\
\hline $\mathrm{BMI}\left(\mathrm{kg} / \mathrm{m}^{2}\right)$ & $29 \cdot 17$ & 6.53 & $26 \cdot 51^{*}$ & 5 \\
\hline $\begin{array}{l}\text { Energy intake (kcal)‡ } \\
\beta \text {-Sitosterol (mg) }\end{array}$ & $1502 \cdot 07$ & $515 \cdot 88$ & $1737 \cdot 10^{\star}$ & 493 \\
\hline $\mathrm{FFQ}$ & $289.30 \dagger$ & $160 \cdot 40$ & $273.40 \dagger$ & 132 \\
\hline $24 \mathrm{HDR}$ & 197.50 & 73.48 & $238 \cdot 10^{\star}$ & 90 \\
\hline \multicolumn{5}{|l|}{ Campesterol (mg) } \\
\hline FFQ & $63.48 \dagger$ & 36.05 & $61 \cdot 82$ & 35 . \\
\hline $24 \mathrm{HDR}$ & $49 \cdot 72$ & 21.52 & $59 \cdot 64^{*}$ & 26 . \\
\hline \multicolumn{5}{|l|}{ Stigmasterol (mg) } \\
\hline FFQ & $59.41 \dagger$ & 41.81 & $54.62 \dagger$ & 37. \\
\hline 24HDR & 39.89 & 27.02 & $44.69^{*}$ & 28. \\
\hline \multicolumn{5}{|l|}{ Other phytosterols $(\mathrm{mg}) \S$} \\
\hline $\mathrm{FFQ}$ & $27 \cdot 49 \dagger$ & $18 \cdot 24$ & $28.05 \dagger$ & 13 \\
\hline 24HDR & 8.47 & $3 \cdot 26$ & $9 \cdot 00^{*}$ & \\
\hline \multicolumn{5}{|l|}{ Total phytosterols $(\mathrm{mg}) \|$} \\
\hline $\mathrm{FFQ}$ & $439.60 \dagger$ & $242 \cdot 20$ & $417.90 \dagger$ & 208. \\
\hline 24HDR & 295.60 & $116 \cdot 40$ & $351.40^{\star}$ & 142 \\
\hline
\end{tabular}

24HDR, 24-h dietary recall.

* Value was significantly different from that for blacks $(P<0.05)$

† Mean value was significantly different from that for $24 \mathrm{HDR}(P<0.05)$.

$\ddagger$ To convert energy in kcal to kJ, multiply by 4.184 .

$\S$ Sum of $\Delta 5+\Delta 7$ avenasterol, avenasterol, brassicasterol, stanols, stigmastanol,

sitostanol, campestanol and unknown.

II Sum of $\beta$-sitosterol, campesterol, stigmasterol and other phytosterols.

Mean plasma concentrations of $\beta$-sitosterol and campesterol in blacks were higher than whites (Table 3). However, statistically significant differences by race were seen only for $\beta$-sitosterol and campesterol. The correlations between plasma sterols $v$. FFQ and 24HDR were 0.02-0.09 and not statistically significant (results not shown).

Unadjusted Pearson correlations between energy-adjusted phytosterol intake in FFQ and 24HDR (Table 4) showed poor to moderate associations $(r 0.15-0.51$ in blacks and $0.10-0.57$ in whites). Overall, de-attenuation improved the correlations of all plant sterol groups in both blacks and whites; however, de-attenuated correlations remained poor for campesterol. All correlations between energy-adjusted phytosterol intake in the FFQ and $24 \mathrm{HDR}$ were statistically significant $(P<0.05)$. Correlations between plant sterols in plasma and plant sterol intake in FFQ or 24HDR were generally poor (below 0.07).

Compared with blacks, whites had higher percentages of exact agreements in all types of named plant sterols but slightly lower in other phytosterols (Table 5). The proportion of exact agreements ranged $27.4-38.6 \%$ in blacks and 30.8-42.3\% in whites. Gross misclassification in blacks was higher than whites, which ranged $4 \cdot 2-9.7 \%$ in blacks and 1.6-11.3\% in whites. Overall, total phytosterols had the highest percentage of exact agreement and the lowest gross misclassification in both blacks and whites

The contribution to total phytosterols by food groups is shown in Fig. 1. On assessment by FFQ, the legumes and soya
Table 3. Average concentration of plasma sterols by race (Mean values and standard deviations)

\begin{tabular}{|c|c|c|c|c|}
\hline \multirow[b]{2}{*}{ Plasma sterol } & \multicolumn{2}{|c|}{ Blacks $(\mu \mathrm{g} / \mathrm{ml})$} & \multicolumn{2}{|c|}{ Whites $(\mu \mathrm{g} / \mathrm{ml})$} \\
\hline & Mean & SD & Mean & SD \\
\hline Plasma sitosterol & 3.75 & 1.87 & $3.06^{*}$ & 1.43 \\
\hline Plasma campesterol & $4 \cdot 87$ & $2 \cdot 63$ & $3.78^{*}$ & 1.96 \\
\hline
\end{tabular}

${ }^{*}$ Mean value was significantly different from that for blacks $(P<0.05)$.

Table 4. Pearson correlations between energy-adjusted phytosterol intake in FFQ and 24-h dietary recall (24HDR) of the Adventist Health Study-2 calibration sub-study by race

\begin{tabular}{|c|c|c|c|c|}
\hline \multirow[b]{2}{*}{ Phytosterol } & \multicolumn{2}{|c|}{ Unadjusted } & \multicolumn{2}{|c|}{ De-attenuated } \\
\hline & Blacks & Whites & Blacks & Whites \\
\hline$\beta$-Sitosterol & $0.51^{\star *}$ & $0.56^{\star \star}$ & $0.67^{\star \star}$ & $0 \cdot 70^{\star *}$ \\
\hline Campesterol & $0.15^{\star}$ & $0 \cdot 10^{*}$ & $0.20^{\star}$ & $0.14^{*}$ \\
\hline Stigmasterol & $0.41^{* *}$ & $0.55^{\star *}$ & $0.58^{\star \star}$ & $0.73^{\text {** }}$ \\
\hline Other phytosterols & $0.32^{\star \star}$ & $0.42^{\star \star}$ & $0.45^{\star \star}$ & $0.56^{\star *}$ \\
\hline Total phytosterols $\ddagger$ & $0.50^{* *}$ & $0.57^{\star *}$ & $0.65^{\star *}$ & $0.72^{\star *}$ \\
\hline
\end{tabular}

Significant correlation between FFQ and 24HDR: ${ }^{*} P<0.05$, ${ }^{\star *} P<0.0001$.

† Sum of $\Delta 5+\Delta 7$ avenasterol, avenasterol, brassicasterol, stanols, stigmastanol, sitostanol, campestanol and unknown.

‡ Sum of $\beta$-sitosterol, campesterol, stigmasterol and other phytosterols.

food group also contributed the greatest proportion (32.61\%), followed by fruits (18.59\%) and fat (17.22 \%), and the olives and avocados food group also contributed the least (1.00\%) to total phytosterols.

\section{Discussion}

Our assessment of the performance of the FFQ in estimating plant sterol intake showed moderate to high correlations when compared with $24 \mathrm{HDR}$ for $\beta$-sitosterol, stigmasterol, other phytosterols and total phytosterols. The correlations that we found on phytosterol consumption are consistent with the previous validation study for a range of nutrients in our and other cohorts $^{(16,31)}$.

The average mean intake of phytosterols from the FFQ was higher than in the $24 \mathrm{HDR}$. It is possible that the FFQ overestimated intake because our FFQ asked about the consumption of over 200 food items which facilitated our study to capture more phytosterol-containing foods than actual intake by the 24HDR. In general, correlations and agreement between the FFQ and 24HDR were higher among whites than blacks.

To our knowledge, only one other group, from Northern Sweden, validated plant sterol intake from an FFQ (with eightyfour food items) with $24 \mathrm{HDR}$ (ten recalls) as a reference ${ }^{(12)}$. In the Northern Sweden study, both crude and de-attenuated correlations were somewhat lower than what we found in AHS-2. In both the Northern Sweden and AHS-2 cohorts, correlations improved after de-attenuation. These findings suggest that both within-person error and energy-adjustment are important components to consider when estimating phytosterol intake.

We note that the definition of 'total phytosterols' by Klingberg et $a l .{ }^{(12)}$ is different from our study. For Klingberg et al. ${ }^{(12)}$, the 
Table 5. Agreement (\%) between the categorisation of energy-adjusted phytosterol intake estimated from FFQ and 24-h dietary recall by race in the Adventist Health Study-2 calibration sub-study participants

\begin{tabular}{|c|c|c|c|c|c|c|c|c|}
\hline \multirow[b]{2}{*}{ Phytosterol } & \multicolumn{4}{|c|}{ Blacks ( $n$ 338) } & \multicolumn{4}{|c|}{ Whites ( $n$ 441) } \\
\hline & Exact & \pm One quartile & \pm Two quartiles & GM‡ & Exact & \pm One quartile & \pm Two quartiles & $\mathrm{GM}^{*}$ \\
\hline$\beta$-Sitosterol & 38.60 & 38.60 & $18 \cdot 30$ & 4.20 & $42 \cdot 30$ & $40 \cdot 70$ & $14 \cdot 30$ & $2 \cdot 70$ \\
\hline Campesterol & 27.40 & 41.00 & $21 \cdot 80$ & $9 \cdot 70$ & $30 \cdot 80$ & 34.80 & $23 \cdot 10$ & $11 \cdot 30$ \\
\hline Stigmasterol & $38 \cdot 10$ & 37.80 & $19 \cdot 20$ & 5.00 & 38.50 & 43.40 & $16 \cdot 50$ & 1.60 \\
\hline Other phytosterols $\dagger$ & 36.60 & $37 \cdot 20$ & 17.70 & 8.60 & 35.80 & $44 \cdot 30$ & 14.70 & $5 \cdot 20$ \\
\hline Total phytosterols $\ddagger$ & $40 \cdot 10$ & $38 \cdot 10$ & $18 \cdot 30$ & 3.50 & 42.50 & 41.40 & 14.50 & 1.60 \\
\hline
\end{tabular}

GM, gross misclassification

* Disagreement by three quartiles.

† Sum of $\Delta 5+\Delta 7$ avenasterol, avenasterol, brassicasterol, stanols, stigmastanol, sitostanol, campestanol and unknown.

$\ddagger$ Sum of $\beta$-sitosterol, campesterol, stigmasterol and other phytosterols.

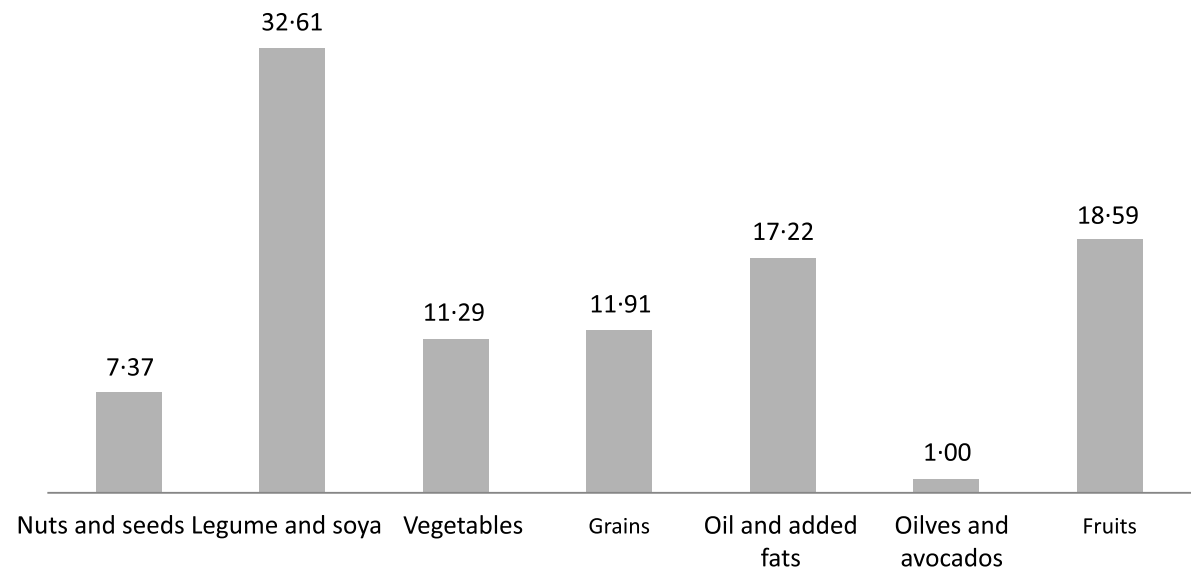

Fig. 1. Percentage contribution to total phytosterol intake by food group from FFQ in the Adventist Health Study-2 calibration sub-study.

total phytosterol category was comprised of five different types of phytosterols, whereas in the AHS-2 calibration sub-study the total included eleven types of phytosterols. The updated comprehensive phytosterol database we compiled in the AHS-2 partly explains the higher estimates observed in our study compared with the Northern Sweden cohort. The relatively higher intake of phytosterols in the AHS-2 also may be driven by the fact that $52 \%$ of the AHS-2 cohort are vegetarian $(28.9 \%$ lacto-ovo vegetarian, $9.8 \%$ pesco vegetarian, $7.6 \%$ vegan and $5.5 \%$ semi vegetarian $)^{(32)}$. Moreover, the wide range of phytosterol intake is a possible reason for the moderately higher correlations in our validation study, which will be beneficial for future diseaserelated hypothesis testing.

We have previously demonstrated the AHS-2 FFQ's ability to discriminate intake of food among individual, particularly foods that contribute to total phytosterol consumption. These food groups included nuts and seeds, legumes and soya, vegetables, grains, oils and added fats, olives and avocado and fruits. Because of this, we examined if the phytosterol concentration in plasma would reflect the wide range of phytosterol intake in our population. We found as others have that correlations of plasma sterol levels with phytosterol intake from either the FFQ or the $24 \mathrm{HDR}$ were poor and not significant. These results confirmed that plasma sterol is not an ideal biomarker of phytosterol intake ${ }^{(33)}$. Phytosterol absorption is less than $2 \%$, whereas cholesterol absorption is up to $60 \%{ }^{(34)}$. The poor absorption of phytosterols is due to its poor substrate for acetyl-CoA acetyltransferase 2 which prevents plant sterols to be packaged into chylomicrons for further circulation throughout the body ${ }^{(35)}$. Phytosterols are returned from the intestinal cells back to gut lumen via the ATP-binding cassette transporters ${ }^{(36)}$. In a study that examine the metabolism of $\beta$-sitosterol and cholesterol in men, Salen et al. further report that cholesterol absorption is inversely correlated with faecal $\beta$-sitosterol ${ }^{(37)}$. Therefore, phytosterol levels in faecal samples could be explored as a possible biomarker of phytosterol intake.

The main contributing food group to total phytosterol intake in both the British diet $(46.96 \%)^{(7)}$ and the Spanish diet $(39.3 \%)^{(8)}$ was the oils food group, whereas in the AHS-2 it was the legumes and soya food group $(32 \cdot 61 \%)$. The proportion of the population following a British diet who consumed plant sterols from added fats (18.32\%) was slightly higher when compared with those in the AHS-2 cohort substudy $(17 \cdot 22 \%)$.

Phytosterol intake from the fruit food group in the present study, particularly as measured by the FFQ (18.59 \%), was greater than in the British diet $(12.7 \%)^{(7)}$. The AHS-2 cohort also had a greater proportion of phytosterol intake from the nuts and seeds food group $(7.37 \%)$ when compared with the British diet $(1.35 \%)^{(7)}$ and the Spanish diet $(2.4 \%)^{(8)}$.

We recognise that our present study has limitations. Lower estimates of the plant sterol intake are greatly influenced by the quality of the database of plant sterol content in foods. We have minimised this effect by compiling the phytosterol content 
in foods from several sources. The first is the USDA SR 27, for phytosterol content in approximately 115 food items, and from other references ${ }^{(5,8,11,12,17-26)}$, for phytosterol content in approximately 189 food items. In addition to deriving phytosterol content from multiple sources, we calculated de-attenuated correlation coefficients which removed the 'noise' of within-person error from $24 \mathrm{HDR}$, and also minimised the influence of total energy intake by using energy-adjusted intake.

\section{Conclusion}

The AHS-2 FFQ is a suitable measurement tool for estimating phytosterol intake in the AHS-2 cohort and may be used to relate intake levels to disease outcomes. Regression calibration will be a necessary step for future studies relating phytosterol intake with an outcome to minimise measurement error in the exposure.

\section{Acknowledgements}

Cohort maintenance was supported by National Institutes of Health/National Cancer Institute grant no. U01CA152939. The phytosterol project was funded by Unilever Research \& Development, Vlaardingen, The Netherlands. The funding agency had no role in the design, analysis or writing of this article.

K. J.-S. designed the research; G. E. F. was the principal investigator of the AHS-2; R. S., A. M. and E. H. generated the phytosterol database; R. S. and A. M. performed statistical analysis; R. S., K. J.-S., G. E. F., C. H. and Y. T.-B. interpreted the results; R. S. wrote the initial draft of the paper; K. J.-S., G. E. F., Y. T.-B. and C. H. critically reviewed and edited the manuscript; R. S. had the primary responsibility for the final content. All authors read and approved the final manuscript. K. J. S. obtained funding from Unilever Research \& Development for this project.

None of the authors has conflicts of interest.

\section{References}

1. Dutta PC (2003) Phytosterols as Functional Food Components and Nutraceuticals. New York: Marcel Dekker.

2. Ryan E, Galvin K, O'Connor TP, et al. (2007) Phytosterol, squalene, tocopherol content and fatty acid profile of selected seeds, grains, and legumes. Plant Foods Hum Nutr 62, 85-91.

3. Lagarda MJ, Garcia-Llatas G \& Farre R (2006) Analysis of phytosterols in foods. J Pharm Biomed Anal 41, 1486-1496.

4. Racette SB, Lin X, Ma L, et al. (2015) Natural dietary phytosterols. J AOAC Int 98, 679-684.

5. Bacchetti T, Masciangelo S, Bicchiega V, et al. (2011) Phytosterols, phytostanols and their esters: from natural to functional foods. Med J Nutrition Metab 4, 165-172.

6. Escurriol V, Cofán M, Moreno-Iribas C, et al. (2010) Phytosterol plasma concentrations and coronary heart disease in the prospective Spanish EPIC cohort. J Lipid Res 51, 618-624.

7. Morton GM, Lee SM, Buss DH, et al. (1995) Intakes and major dietary sources of cholesterol and phytosterols in the British diet. J Hum Nutr Diet 8, 429-440.
8. Jimenez-Escrig A, Santos-Hidalgo AB \& Saura-Calixto F (2006) Common sources and estimated intake of plant sterols in the Spanish diet. J Agric Food Chem 54, 3462-3471.

9. AbuMweis SS, Barake R \& Jones PJH (2008) Plant sterols/ stanols as cholesterol lowering agents: a meta-analysis of randomized controlled trials. Food Nutr Res 52. doi: 10.3402/ fnr.v52i0.1811

10. Tapiero H, Townsend DM \& Tew KD (2003) Phytosterols in the prevention of human pathologies. Biomed Pharmacother 57, 321-325.

11. Weihrauch JL \& Gardner JM (1978) Sterol content of foods of plant origin. J Am Diet Assoc 73, 39-47.

12. Klingberg S, Winkvist A, Hallmans G, et al. (2013) Evaluation of plant sterol intake estimated with the Northern Sweden FFQ. Public Health Nutr 16, 460-467.

13. Butler TL, Fraser GE, Beeson WL, et al. (2008) Cohort profile: the Adventist Health Study-2 (AHS-2). Int J Epidemiol 37, $260-265$

14. Orlich MJ, Singh PN, Sabaté J, et al. (2013) Vegetarian dietary patterns and mortality in adventist health study 2. JAMA Intern Med 173, 1230-1238.

15. Willett W (2012) Nutritional Epidemiology, 3rd ed. New York: Oxford University Press.

16. Jaceldo-Siegl K, Knutsen SF, Sabaté J, et al. (2010) Validation of nutrient intake using an FFQ and repeated $24 \mathrm{~h}$ recalls in black and white subjects of the Adventist Health Study-2 (AHS-2). Public Health Nutr 13, 812-819.

17. Phillips KM, Ruggio DM \& Ashraf-Khorassani M (2005) Phytosterol composition of nuts and seeds commonly consumed in the United States. J Agric Food Chem 53, 9436-9445.

18. Normén L, Bryngelsson S, Johnsson M, et al. (2002) The phytosterol content of some cereal foods commonly consumed in Sweden and in The Netherlands. J Food Compos Anal 15, 693-704.

19. Piironen V, Toivo J, Puupponen-Pimiä R, et al. (2003) Plant sterols in vegetables, fruits and berries. J Sci Food Agric 83, 330-337.

20. Normén L, Ellegård L, Brants H, et al. (2007) A phytosterol database: fatty foods consumed in Sweden and The Netherlands. J Food Compos Anal 20, 193-201.

21. Normén L, Johnsson M, Andersson H, et al. (1999) Plant sterols in vegetables and fruits commonly consumed in Sweden. Eur J Nutr 38, 84-89.

22. Piironen V, Lindsay DG, Miettinen TA, et al. (2000) Plant sterols: biosynthesis, biological function and their importance to human nutrition. J Sci Food Agric 80, 939-966.

23. Ferrari RA, Esteves W, Mukherjee KD, et al. (1997) Alteration of sterols and steryl esters in vegetable oils during industrial refining. J Agric Food Chem 45, 4753-4757.

24. Awad AB, Chan KC, Downie AC, et al. (2000) Peanuts as a source of beta-sitosterol, a sterol with anticancer properties. Nutr Cancer 36, 238-241.

25. Phillips KM, Ruggio DM, Toivo JI, et al. (2002) Free and esterified sterol composition of edible oils and fats. J Food Compos Anal 15, 123-142.

26. Chandra Dutta P \& Appelqvist L-Å (1996) Saturated sterols (Stanols) in unhydrogenated and hydrogenated edible vegetable oils and in cereal lipids. J Sci Food Agric 71, 383-391.

27. US Department of Agriculture, A.R.S., Beltsville Human Nutrition Research Center, and N.D. Laboratory (2014) Composition of foods raw, processed, prepared. USDA National Nutrient Database for Standard Reference, Release 27, p. 25.

28. Chan J, Knutsen SF, Sabate J, et al. (2007) Feasibility of running clinics to collect biological specimens in a Nationwide Cohort Study-Adventist Health Study-2. Ann Epidemiol 17, 454-457. 
29. Lütjohann D, Björkhem I, Beil UF, et al. (1995) Sterol absorption and sterol balance in phytosterolemia evaluated by deuteriumlabeled sterols: effect of sitostanol treatment. J Lipid Res 36, 1763-1773.

30. Jaceldo-Siegl K, Fan J, Sabaté J, et al. (2011) Race-specific validation of food intake obtained from a comprehensive FFQ: the Adventist Health Study-2. Public Health Nutr 14, 1988-1997.

31. Midthune D, Schatzkin A, Subar AF, et al. (2011) Validating an FFQ for intake of episodically consumed foods: application to the National Institutes of Health-AARP Diet and Health Study. Public Health Nutr 14, 1212-1221.

32. Orlich MJ, Singh PN, Sabaté J, et al. (2013) Vegetarian dietary patterns and mortality in Adventist Health Study 2. JAMA Intern Med 173, 1230-1238.
33. Plat J \& Mensink RP (2005) Plant stanol and sterol esters in the control of blood cholesterol levels: mechanism and safety aspects. Am J Cardiol 96, Suppl. 1, 15-22.

34. Rozner S \& Garti N (2006) The activity and absorption relationship of cholesterol and phytosterols. Colloids Surf $A$ Physicochem Eng Asp 282-283, Suppl. C, 435-456.

35. Jakulj L, Mohammed H, van Dijk TH, et al. (2013) Plasma plant sterols serve as poor markers of cholesterol absorption in man. J Lipid Res 54, 1144-1150.

36. Trautwein EA \& Demonty I (2007) Phytosterols: natural compounds with established and emerging health benefits. Oilseeds Fats Crops Lipids 14, 259-266.

37. Salen G, Ahrens EH Jr \& Grundy SM (1970) Metabolism of $\beta$-sitosterol in man. J Clin Invest 49, 952-967. 